\title{
FACTORES ASOCIADOS A LA APARICIÓN DE DEFECTOS DE DESARROLLO DEL ESMALTE EN DENTICIÓN DECIDUA, EN EL HOSPITAL LUIS HEYSEN INCHÁUSTEGUI, CHICLAYO, 2019
}

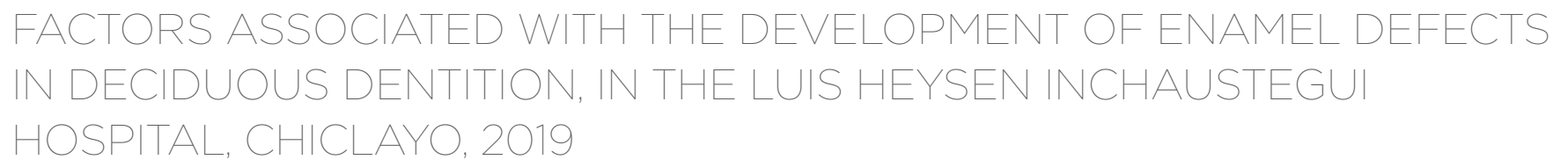

Karen Lizeth Serrano-Arrasco ${ }^{*}$ (1) karenlizeth_sa90@hotmail.com

Artículo recibido: 02/01/2020

Arbitrado por pares

Artículo aceptado: 03/03/2020

* Autor corresponsal:

Karen Lizeth Serrano-Arrasco karenlizeth_sa90@homail.com

Citar como: Serrano-Arrasco K. Factores asociados a la aparición de defectos de desarrollo del esmalte en dentición decidua, en el hospital Luis Heysen Incháustegui, Chiclayo, 2019. Rev Cient Odontol (Lima) 2020; 8(1): e005.

DOI: $10.21142 / 2523-2754-0801-2020-005$

\section{RESUMEN}

Objetivo: Identificar los factores asociados a la presencia de los defectos de desarrollo del esmalte (DDE) de la dentición decidua. Material y métodos: El diseño de investigación fue, observacional, prospectivo y transversal; la muestra fue de 135 niños, 37 cumplieron los criterios de inclusión para el estudio, todos fueron evaluados en un consultorio dental. Resultados: Se realizó el análisis estadístico de los datos de manera descriptiva, con (chi-cuadrado, coeficiente de contingencia y gamma) para encontrar y evaluar las relaciones, respecto al DDE. E1 59\% de los niños evaluados fueron de sexo masculino, mientras que el $41 \%$ fueron niñas; en todos se encontró opacidad difusa. El nacimiento pretérmino se presentó en un 30\% de los casos estudiados, mientras que el 70\% cumplió el ciclo normal. La opacidad difusa fue la más prevalente. E1 27\% que nació con bajo peso presentan DDE. Se reportaron 4 niños con hipoplasia, de los cuales todos nacieron con un peso normal (>2500 gramos), solo 10 niños del total tuvieron un bajo peso, de entre 1500 y 2500 gramos y la mayoría fueron afectados por una opacidad difusa. E1 78\% de los niños presentó DDE en la zona anterior de la dentición. Conclusión: Los factores asociados en la aparición de DDE en el presente estudio no sería ninguno de los factores citados; sin embargo, el análisis descriptivo desarrollado establece que el sexo del bebé, el peso al nacer, el tipo de parto, las infecciones maternas durante la gestación y las infecciones durante el primer año de vida del bebé, podrían influenciar.

Palabras clave: Defecto de desarrollo de esmalte, factor asociado, dentición decidua

\begin{abstract}
Objective: To identify the factors associated with the presence of enamel development defects (DDE) of deciduous teeth. Material and methods: This was a descriptive, prospective, transversal, observational study. The sample included 135 children, 37 of whom met the inclusion and exclusion criteria of the study and all were evaluated in a dental office. Results: Descriptive statistical analysis of the data was performed with the chi-square test, contingency coefficients and gamma statistics to determine and evaluate the relationships with respect to DDE. 59\% of the children evaluated were male while $41 \%$ were females, with all showing diffuse opacity. Preterm birth occurred in $30 \%$ of the cases studied, while $70 \%$ had been delivered at term. Of the children with DDE, $27 \%$ were underweight at birth. Four children with hypoplasia had had a normal weight at birth $(>2500$ grams), while only 10 children had a low weight (1500-2500 g), and most presented diffuse opacity. $78 \%$ of children had DDE in the previous teething area. Conclusion: The factors associated with the occurrence of DDE in this study were not related to any of the factors cited; however sex, birth weight, type of delivery, maternal infections during pregnancy and infections during the first year of the baby's life may influence the presence of DDE.
\end{abstract}

Keywords: Enamel development defect, associated factor, deciduous teeth.

\footnotetext{
1 Especialidad de Odontopediatría, Escuela de Odontología, Universidad Católica Santo Toribio de Mogrovejo. Chiclayo, Perú.
} 


\section{INTRODUCCIÓN}

Los defectos de desarrollo del esmalte (DDE) en dentición decidua son alteraciones de la matriz o la mineralización de los tejidos duros dentarios durante el periodo de la amelogénesis; además, reconocemos como un factor asociado a aquel elemento que puede condicionar una situación y volverse causante de la evolución o transformación de los hechos $\left(^{1}\right)$.

Los DDE se consideran como un factor que predispone una alta prevalencia de caries dental, pues la hipocalcificación y la hipoplasia del esmalte muestran mayor porosidad y contribuyen al aumento de la retención de placa bacteriana, es por eso que debemos diagnosticarlos a tiempo y darles un tratamiento adecuado. Los DDE podrían ser el resultado de factores sistémicos, genéticos o ambientales, como el nacimiento pretérmino, el bajo peso al nacer, las infecciones, la desnutrición o los trastornos metabólicos, muchos de los cuales tienen una mayor incidencia en familias de bajo nivel socioeconómico. Se sabe que el esmalte dental no puede remodelarse, y los defectos que ocurren en este presentan un registro de los daños sufridos por el órgano del esmalte durante su desarrollo. Sin embargo, determinar el momento exacto de las alteraciones en el esmalte en desarrollo es a menudo difícil, debido a la falta de conocimiento sobre la cronología de las diferentes etapas de la amelogénesis, así como la diferencia en cada individuo respecto de las tasas de formación de esmalte $\left(^{1,2}\right)$.

Como consecuencia de lo expuesto, se planteó en la investigación el siguiente problema: ¿cuáles fueron los factores asociados con la aparición de defectos en el desarrollo del esmalte en dentición decidua, en el hospital Luis Heysen Inchaústegui (Chiclayo), en 2019?

Ante la interrogante, se formuló el siguiente objetivo general: identificar los factores asociados con la aparición de DDE en dentición decidua, en el hospital Luis Heysen Incháustegui (Chiclayo), en 2019.

Así mismo, se plantearon los siguientes objetivos específicos: determinar la aparición de DDE en dentición decidua según sexo, determinar la aparición de DDE en dentición decidua según localización, determinar la aparición de DDE en dentición decidua según tipo de nacimiento, determinar la aparición de DDE en dentición decidua según peso al nacer, determinar la aparición de DDE en dentición decidua según factores prenatales y determinar la aparición de DDE en dentición decidua según factores postnatales.

Como hipótesis se tiene que existen factores asociados con la aparición de DDE en dentición decidua en el hospital Luis Heysen Incháustegui (Chiclayo), en 2019.

\section{MATERIALES Y MÉTODOS}

Se realizó un estudio observacional prospectivo y transversal, una vez aprobado el proyecto de tesis por el Comité de Ética en Investigación de la Facultad de Medicina de la Universidad Santo Toribio de Mogrovejo, y con la autorización por parte del Hospital.

Se coordinó con el jefe del servicio de Odontología del hospital Luis Heysen Incháustegui para proceder a la evaluación de los niños, así que, de un total de 135 pacientes, luego de la selección según criterios de inclusión y exclusión, se encontró a 37 niños con DDE, a cuyas madres se les explicó el procedimiento y se les hizo firmar el consentimiento informado. Los niños fueron evaluados en la unidad dental y el operador utilizó medidas de bioseguridad como gorro, mascarilla y guantes de látex.

Se evaluaron las piezas dentarias, empezando por el cuadrante V. Se revisó las superficies vestibulares, oclusal y palatina, con espejo bucal y luz artificial. Las superficies fueron limpiadas con agua y gasa, y observadas secas para visualizar los posibles defectos. Se anotaron los hallazgos en la ficha que contiene el índice de DDE y, posteriormente, se realizó una entrevista estructurada a la madre para evaluar los posibles factores relacionados con la aparición de DDE.

Los datos se gestionaron en una base de datos de Microsoft Office Excel 2016 (Microsoft Corporation, Washington, EE. UU.). Luego de verificar las concordancias y hacer el control de calidad, se exportaron al programa estadístico 
Tabla 1. Tipo de defecto según el sexo del paciente. Hospital Luis Heysen Incháustegui (Chiclayo), 2019

\begin{tabular}{|c|c|c|c|c|c|c|}
\hline & \multicolumn{4}{|c|}{ Defecto del esmalte } & \multirow[b]{2}{*}{ Total } \\
\hline & & $\begin{array}{l}\text { Opacidad } \\
\text { demarcada }\end{array}$ & $\begin{array}{l}\text { Opacidad } \\
\text { difusa }\end{array}$ & Hipoplasia & Combinada & \\
\hline \multirow{2}{*}{$\begin{array}{l}\text { Sexo del } \\
\text { paciente }\end{array}$} & Femenino & 4 & 8 & 2 & I & 15 \\
\hline & Masculino & 5 & 14 & 2 & 1 & 22 \\
\hline Total & & 9 & 22 & 4 & 2 & 37 \\
\hline
\end{tabular}

Tabla 2. Tipo de defecto según el tipo de nacimiento de niño. Hospital Luis Heysen Incháustegui (Chiclayo), 2019

\begin{tabular}{|c|c|c|c|c|c|c|}
\hline & & \multicolumn{4}{|c|}{ Defecto del esmalte } & \multirow[b]{2}{*}{ Total } \\
\hline & & $\begin{array}{l}\text { Opacidad } \\
\text { demarcada }\end{array}$ & $\begin{array}{c}\text { Opacidad } \\
\text { difusa }\end{array}$ & Hipoplasia & Combinada & \\
\hline \multirow{2}{*}{$\begin{array}{l}\text { Tipo de } \\
\text { nacimiento }\end{array}$} & Término & 4 & 17 & 4 & I & 26 \\
\hline & Pretérmino & 5 & 5 & 0 & I & II \\
\hline Total & & 9 & 22 & 4 & 2 & 37 \\
\hline
\end{tabular}

SPSS en español (versión 25.0, PASW Statistics Chicago, EE. UU.), mediante el cual se obtuvo el análisis descriptivo de todas las variables con tablas y gráficos de frecuencias. Además, se realizaron análisis relacionales o de asociación con las pruebas Chi-cuadrado de Pearson, coeficientes de contingencia y gamma, aplicadas en las variables de cada objetivo.

\section{RESULTADOS}

Se encontró que el 59\% de los niños evaluados eran de sexo masculino, mientras que el $41 \%$ eran niñas. De los 22 niños registrados, 14 tienen opacidad difusa; por otro lado, de las 15 niñas, 8 tuvieron el mismo tipo de defecto.

El nacimiento pretérmino se presentó en un 30\% de los casos, mientras que el $70 \%$ cumplió el ciclo normal. La opacidad difusa fue la más prevalente, pues se presentó en 17 niños con nacimiento a término y en 5 niños con nacimiento pretérmino.

Del total de niños que presentaron DDE, el 27\% nació con bajo peso, como consecuencia de un nacimiento pretérmino. Se reportaron 4 niños con hipoplasia, de los cuales todos nacieron con un peso normal ( $>2500$ gramos). Solo 10 niños del total tuvieron bajo peso (entre
Tabla 3. Tipo de defecto según el peso al nacer del niño. Hospital Luis Heysen Incháustegui (Chiclayo), 2019

\begin{tabular}{|c|c|c|c|c|c|c|}
\hline & \multicolumn{4}{|c|}{ Defecto del esmalte } & \multirow[b]{2}{*}{ Total } \\
\hline & & $\begin{array}{l}\text { Opacidad } \\
\text { demarcada }\end{array}$ & $\begin{array}{l}\text { Opacidad } \\
\text { difusa }\end{array}$ & Hipoplasia & Combinada & \\
\hline \multirow{2}{*}{$\begin{array}{l}\text { Peso al } \\
\text { nacer }\end{array}$} & $\begin{array}{l}\text { Bajo peso: } \\
\text { I500-2500 }\end{array}$ & 4 & 5 & 0 & । & 10 \\
\hline & $\begin{array}{l}\text { Peso normal: } \\
>2500\end{array}$ & 5 & 17 & 4 & । & 27 \\
\hline Total & & 9 & 22 & 4 & 2 & 37 \\
\hline
\end{tabular}

Tabla 4. Tipo de defecto según la edad de la madre del niño al nacer. Hospital Luis Heysen Incháustegui (Chiclayo) 2019

\begin{tabular}{|c|c|c|c|c|c|c|}
\hline & \multicolumn{6}{|c|}{ Defecto del esmalte } \\
\hline & & $\begin{array}{l}\text { Opacidad } \\
\text { demarcada }\end{array}$ & $\begin{array}{c}\text { Opacidad } \\
\text { difusa }\end{array}$ & Hipoplasia & Combinada & Total \\
\hline \multirow{3}{*}{$\begin{array}{l}\text { Edad } \\
\text { materna }\end{array}$} & $<18$ & 0 & I & 0 & 0 & I \\
\hline & $18-34$ & 7 & 20 & 2 & I & 30 \\
\hline & 35 a más & 2 & I & 2 & । & 6 \\
\hline \multicolumn{2}{|l|}{ Total } & 9 & 22 & 4 & 2 & 37 \\
\hline
\end{tabular}

Tabla 5. Tipo de defecto según la existencia de infecciones maternas o infecciones en el proceso de gestación del niño. Hospital Luis Heysen Incháustegui (Chiclayo), 2019

\begin{tabular}{|c|c|c|c|c|c|c|}
\hline & \multicolumn{6}{|c|}{ Defecto del esmalte } \\
\hline & & $\begin{array}{l}\text { Opacidad } \\
\text { demarcada }\end{array}$ & $\begin{array}{c}\text { Opacidad } \\
\text { difusa }\end{array}$ & Hipoplasia & Combinada & Total \\
\hline \multirow{2}{*}{$\begin{array}{l}\text { Infecciones } \\
\text { maternas }\end{array}$} & Sí & 3 & 13 & 2 & 2 & 20 \\
\hline & No & 6 & 9 & 2 & 0 & 17 \\
\hline Total & & 9 & 22 & 4 & 2 & 37 \\
\hline
\end{tabular}

1500 y 2500 gramos) y la mayoría fueron afectados por una opacidad difusa.

Del total de casos evaluados, el 2,7\% tenía una madre menor de 18 años; el 81,1\%, una madres de entre 18 y 34 años; y el 16,2\%, madres mayores a 35 años. Solo una madre menor de 18 años tuvo un niño con opacidad difusa, mientras que las madres con más de 35 años tuvieron 2 niños con hipoplasia y opacidad demarcada. Por otro lado, en 30 madres de entre 18 y 34 años el defecto más prevalente fue la opacidad difusa.

En el 54\% de los casos estudiados, las madres sufrieron algún tipo de infección durante el periodo gestacional, lo que resulta un factor importante, ya que puede afectar el ciclo de desarrollo de la dentición de los niños. 
Tabla 6. Tipo de defecto según el uso de incubadora por parte del niño al nacer. Hospital Luis Heysen Incháustegui (Chiclayo), 2019

\begin{tabular}{|c|c|c|c|c|c|c|}
\hline & \multicolumn{6}{|c|}{ Defecto del esmalte } \\
\hline & & $\begin{array}{l}\text { Opacidad } \\
\text { demarcada }\end{array}$ & $\begin{array}{c}\text { Opacidad } \\
\text { difusa }\end{array}$ & Hipoplasia & Combinada & Total \\
\hline \multirow{2}{*}{ Incubadora } & Sí & 0 & 5 & 0 & I & 6 \\
\hline & No & 9 & 17 & 4 & 1 & 31 \\
\hline Total & & 9 & 22 & 4 & 2 & 37 \\
\hline
\end{tabular}

Tabla 7. Tipo de defecto según la existencia de infecciones en el primer año de vida del niño. Hospital Luis Heysen Incháustegui (Chiclayo), 2019

\begin{tabular}{|c|c|c|c|c|c|c|}
\hline & & \multicolumn{4}{|c|}{ Defecto del esmalte } & \multirow[b]{2}{*}{ Total } \\
\hline & & $\begin{array}{l}\text { Opacidad } \\
\text { demarcada }\end{array}$ & $\begin{array}{l}\text { Opacidad } \\
\text { difusa }\end{array}$ & Hipoplasia & Combinada & \\
\hline \multirow{2}{*}{$\begin{array}{l}\text { Infecciones } \\
\text { durante el } \\
\text { primer año } \\
\text { de vida }\end{array}$} & Sí & 6 & 11 & I & I & 19 \\
\hline & No & 3 & 11 & 3 & । & 18 \\
\hline Total & & 9 & 22 & 4 & 2 & 37 \\
\hline
\end{tabular}

Tabla 8. Zona afectada según el tipo de defecto del niño al nacer. Hospital Luis Heysen Incháustegui (Chiclayo), 2019

\begin{tabular}{llccc} 
& & \multicolumn{2}{c}{ Localización } & Total \\
& Opacidad demarcada & 8 & 1 & 9 \\
\hline \multirow{2}{*}{ Defecto del } & Posterior & \\
esmalte & Opacidad difusa & 18 & 4 & 22 \\
& Hipoplasia & 3 & 1 & 4 \\
\hline Total & Combinada & 0 & 2 & 2 \\
\hline
\end{tabular}

De los 37 niños con DDE, el 16\% fue puesto en incubadora, lo cual se relacionó con los niños que nacieron a pretérmino. En este reporte, presentamos la relación entre el uso de incubadora al momento de nacimiento, como asociación con algún tipo de DDE. Se encontró que solo hubo 5 casos de opacidad.

Según el reporte, ninguno de los niños tuvo alteraciones respiratorias al momento de nacer, por lo que no podríamos determinar si la intubación puede causar un defecto de esmalte o alterar la normalidad en el desarrollo del órgano dental.

El 51\% de los niños sufrió estas infecciones. De los 19 casos de niños que experimentaron infecciones en
Tabla 9. Resultados de los test aplicados para probar s algún factor está relacionado con el defecto del paciente - la localización de su defecto

\begin{tabular}{|c|c|c|c|c|}
\hline $\begin{array}{c}\text { Prueba } \\
\text { estadística }\end{array}$ & Variables & $\begin{array}{c}\text { Valor } \\
\text { estadístico }\end{array}$ & Significancia & Decisión \\
\hline $\begin{array}{l}\text { Coeficiente de } \\
\text { contingencia }\end{array}$ & $\begin{array}{l}\text { Sexo del paciente vs. } \\
\text { defecto de esmalte }\end{array}$ & 0,108 & 0,932 & $\begin{array}{l}\text { No existe } \\
\text { relación. }\end{array}$ \\
\hline $\begin{array}{l}\text { Coeficiente } \\
\text { de } \\
\text { contingencia }\end{array}$ & $\begin{array}{l}\text { Tipo de nacimiento } \\
\text { vs. defecto de } \\
\text { esmalte }\end{array}$ & 0,359 & 0,14 & $\begin{array}{l}\text { No existe } \\
\text { relación. }\end{array}$ \\
\hline $\begin{array}{l}\text { Coeficiente de } \\
\text { contingencia }\end{array}$ & $\begin{array}{l}\text { Peso al nacer vs. } \\
\text { defecto de esmalte }\end{array}$ & 0,298 & 0,307 & $\begin{array}{l}\text { No existe } \\
\text { relación. }\end{array}$ \\
\hline $\begin{array}{l}\text { Coeficiente } \\
\text { de } \\
\text { contingencia }\end{array}$ & $\begin{array}{l}\text { Edad materna vs. } \\
\text { defecto de esmalte }\end{array}$ & 0,42 & 0,242 & $\begin{array}{l}\text { No existe } \\
\text { relación. }\end{array}$ \\
\hline $\begin{array}{l}\text { Coeficiente de } \\
\text { contingencia }\end{array}$ & $\begin{array}{l}\text { Infecciones maternas } \\
\text { vs. defecto de } \\
\text { esmalte }\end{array}$ & 0,294 & 0,32 & $\begin{array}{l}\text { No existe } \\
\text { relación. }\end{array}$ \\
\hline $\begin{array}{l}\text { Coeficiente } \\
\text { de } \\
\text { contingencia }\end{array}$ & $\begin{array}{l}\text { Uso de incubadora } \\
\text { vs defecto de } \\
\text { esmalte }\end{array}$ & 0,341 & 0,181 & $\begin{array}{l}\text { No existe } \\
\text { relación. }\end{array}$ \\
\hline $\begin{array}{l}\text { Coeficiente de } \\
\text { contingencia }\end{array}$ & $\begin{array}{l}\text { Infecciones durante } \\
\text { el primer año de } \\
\text { vida vs. defecto de } \\
\text { esmalte }\end{array}$ & 0,225 & 0,578 & $\begin{array}{l}\text { No existe } \\
\text { relación. }\end{array}$ \\
\hline $\begin{array}{l}\text { Chi-cuadrado } \\
\text { de Pearson }\end{array}$ & $\begin{array}{l}\text { Defecto del esmalte } \\
\text { vs. Localización del } \\
\text { defecto }\end{array}$ & 8,017 & 0,046 & $\begin{array}{l}\text { Existe } \\
\text { relación. }\end{array}$ \\
\hline
\end{tabular}

su primer año de vida, 11 tuvieron opacidad difusa, la cual fue prevalente en aquellos que no experimentaron infecciones en su primer año de vida (11 casos).

En nuestra investigación, el 78\% de los niños presentó DDE en la zona anterior de la dentición. Del total de casos estudiados, 18 de ellos con opacidad difusa se localizaron en la parte anterior. Hubo un único caso de opacidad demarcada ubicado en la parte posterior; 3 casos de hipoplasia en la parte anterior, mientras que los 2 casos con defecto combinado se localizaron en la parte posterior.

\section{DISCUSIÓN}

La literatura y numerosos estudios han evidenciado que existen diversos factores asociados con la aparición de defectos de desarrollo del esmalte, ya que las piezas dentarias inician su formación en los primeros meses de vida intrauterina, y cualquier pertubación durante este periodo podría alterar las células formadoras de esmalte, 
con la consecuencia de alguna alteración estructural del esmalte.

En nuestro estudio, se consideró sexo, tipo de nacimiento, peso al nacer y factores prenatales como edad materna e infecciones maternas durante la gestación y factores postnatales como uso de incubadora en el nacimiento, intubación endotraqueal e infecciones durante el primer año de vida. Así mismo, evidenciamos que la zona más afectada con la aparción de DDE es la zona anterior, con los incisivos primarios como los predominantes.

Encontramos que el $59 \%$ de los niños evaluados eran de sexo masculino, mientras que el $41 \%$ eran niñas. A partir de esto, podemos concluir que la mayoría de niños son propensos a desarrollar DDE, tal como se registra en los estudios de Basha (2014), Wong (2014), Masumo (2013) y Massoni (2009) (1,4-6).

La opacidad difusa fue la más prevalente y se presentó en 17 niños con nacimiento a término, y en 5 niños que tuvieron un nacimiento pretérmino; sin embargo, Wagner (2017) determinó que el nacimiento pretérmino y la hospitalización en el primer año de vida pueden considerarse como un factor de riesgo para la presencia de DDE. Así mismo, la revisión sistemática de Jacobsen et al. (2014) sostiene que el nacimiento pretérmino es un factor que contribuye a la aparición de hipoplasias en dentición primaria.Además de los 37 niños referenciados, el 16\% fue puesto en incubadora, lo cual se relacionó con los niños que nacieron a pretérmino; por otro lado, ningún niño recibió intubación endotraqueal, la cual es considerada, según la literatura, como factor de riesgo para la aparición de DDE y problemas estructurales del paladar $\left({ }^{3,4}\right)$.

Se reportaron 10 niños con bajo peso al nacer, entre 1500 y 2500 gramos, y la mayoría fueron afectados por opacidad difusa. Además, de todos los que nacieron con un peso normal (> 2500 gramos), se encontraron 17 niños con opacidad difusa, es así como Jacobsen et al. (2014), en su revisión sistemática, concluyó que existe una asociación entre el peso al nacer menor a 1500 gr y el riesgo de aparición de opacidades en el esmalte en dientes primarios.
Por otro lado, consideramos analizar la edad materna, como lo hicieron Wong (2014), Masumo (2013) y Masson (2009) $(1,6,7)$. Observamos que solo una madre menor de 18 años tuvo un niño con opacidad difusa, mientras que las madres con más de 35 años tuvieron 2 niños con hipoplasia y opacidad demarcada. Por otro lado, en 30 madres de entre 18 y 34 años el defecto más prevalente fue la opacidad difusa. En cuanto a las infecciones maternas, en el $54 \%$ de los casos estudiados, las madres sufrieron algún tipo de infección durante el periodo gestacional, lo que resulta un factor importante, ya que puede afectar el ciclo de desarrollo de la dentición en los niños.

Así mismo, muchos factores prenatales pueden influir en el desarrollo de los dientes primarios y, posiblemente, los permanentes, como la salud materna, la ingesta de medicamentos, el tabaquismo y el consumo de alcohol $\left({ }^{4}\right)$. Se presenta también una distribución de los tipos de defectos encontrados según la infecciones durante el periodo de gestación, pues de 20 madres que sufrieron algún tipo de infección solo 13 niños tuvieron opacidad difusa, mientras que 9 de las 17 madres que no sufrieron complicaciones durante el periodo de gestación tuvieron niños con opacidad difusa.

E1 51\% de los niños sufrió alguna infección durante el primer año de vida y 11 tuvieron opacidad difusa. Tal como lo evidencia Masumo (2013), la situación socioeconómica de la familia y las enfermedades infecciosas de la primera infancia se han asociado con un aumento de defectos de esmalte en dentición primaria. De la misma manera, Wong (2014) manifiesta que los niños con enfermedades graves en la vida temprana fueron casi 8 veces más propensos que los niños sanos a ser afectados por DDE $(1,6)$.

Finalmente, estudiamos la zona más afectada en nuestra investigación. En el 78\% del total de casos estudiados, los DDE se presentaron en la zona anterior de la dentición, 18 de ellos con opacidad difusa. Se presentó un único caso de opacidad demarcada en la parte posterior, 3 casos de hipoplasia en la parte anterior, mientras que los 2 casos con defecto combinado se localizaron en la parte posterior. Esto se confirma por el estudio de Massoni (2009), el cual también halló alteraciones 
de esmalte principalmente en los incisivos centrales superiores. Así mismo, Masumo (2013) concluye que los dientes afectados con mayor frecuencia fueron los incisivos centrales superiores (29,0\%-30,5\%), por lo que los DDE son un factor de riesgo para lesiones de caries dental, ya que comprometen la calidad y cantidad de esmalte dentario; lo cual tiene implicancias estéticas, complicaciones como sensibilidad dentaria y fracturas dentarias, esto repercute en la calidad de vida de los niños $\left.{ }^{(6,7}\right)$.

\section{CONCLUSIONES}

Mediante los test estadísticos utilizados para encontrar los factores asociados a los DDE, no se encontró evidencia de que alguno influyera determinantemente en la aparición de DDE.

- Según el sexo del paciente, encontramos que el género más afectado fue el masculino, con el 59\% de casos; sin embargo, el análisis estadístico no encontró influencia de esta variable sobre el defecto.

- La zona anterior fue la más afectada y la opacidad difusa fue el DDE más prevalente.

- Respecto del tipo de nacimiento, no fue un factor determinante para la presencia de DDE.
- Con el peso como variable posiblemente asociada al DDE, el análisis realizado determinó no ser influyente o estar asociado.

- La no existencia de infecciones maternas, la ausencia de infecciones durante el primer año de vida y el no usar incubadora generan predisposición a presentar defectos de hipoplasia, y las infecciones maternas, las infecciones en el bebé y el uso de incubadora podrían ser condicionantes de la opacidad difusa.

- El haber padecido infecciones maternas durante la etapa de gestación, y durante el primer año de vida, puede traer como consecuencia la presencia de DDE en la parte anterior de la dentición.

- Los factores posnatales estudiados no resultaron ser influyentes en la aparición del DDE, esto puede deberse a la poca cantidad de la muestra.

Contribución de autoría: Karen Lizeth SerranoArrasco ha participado en la elaboración del artículo, la recolección de la información, la redacción y aprobación de la versión final.

Fuente de financiamiento: Autofinanciado.

Potenciales conflictos de interés: La autora declara no tener ningún tipo de conflicto de interés. 


\section{REFERENCIAS BIBLIOGRÁFICAS}

1. Wong HM, Peng SM, Wen YF, King NM, McGrath CP. Risk factors of developmental defects of enamel - A prospective cohort study. Plos One 2014; 9 (10): e109351.

2. Seow W. Etiology of developmental enamel defects in the primary dentition. Clin Dent Review. 2017; 1 (7): 1-8.

3. Wagner Y. Developmental defects of enamel in primary teeth, findings of a regional German birth cohort study. BMC Oral Health. 2017; 17 (10): 1-8. doi: 10.1186/s12903-016-0235-7

4. Jacobsen P, Heriksen T, Haubek D, Ostergaard J. Developmental enamel defects in children prenatally exposed to anti-epileptic drugs. Plos One 2013; 8 (3): 1-6. doi: 10.1371/journal. pone.0058213

5. Basha S, Mohamed R, Swamy H. Prevalence and associated factors to developmental defects of enamel in primary and permanent dentition. Oral Health Dent Manang. 2014; 13 (3): 588-94.

6. Masumo R, Bardsen A, Astrom A. Developmental defects of enamel in primary teeth and association with early life course events: a studdy of 6-36 month old children in Manyara, Tanzania. BMC Oral Health 2013; 13 (21): 1-11.

7. Massoni A, Chávez A, Rosemblatt A, Sampaio F, Oliveira A. Prevalence of enamel defects related to pre, peri- and postnatal factors in a Brazilian population. Community Dent Health 2009; 26 (3): 143-9. doi: 10.1922/CDH_2268Massoni07

8. Nelson S, Albert JM, Lombardi G, Wishnek S, Assad G, Kirchner H, et al. Dental caries and enamel defects in very low birth weight adolecents. Caries Res. 2010; 44: (6): 509-18.

9. Robles M. Estudio y prevalencia de los defectos de desarrollo del esmalte en la población infantil granadina [Tesis doctoral]. Granada: Universidad de Granada; 2010.

10. Naranjo M. Terminología, clasificación y medición de los defectos en el desarrollo del esmalte. Revista Javeriana 2013; 32 (68): 33-4.

11. Osorio J. Prevalencia de defectos de desarrollo del esmalte en dentición temporal en niños de 4 a 6 años que asisten al colegio instituto pedagógico Arturo Ramírez Montúfar (IPARM) [Tesis de grado]. Bogotá: Universidad Nacional de Colombia; 2012.

12. Salinitri S, Seow W. Developmental enamel defects in the primary detition: aetiology and clinical management. Aust Dent J. 2013; 58: 133-40. doi: 10.1111/adj.12039
13. Wong H. Aetiological factors for developmental defects of enamel. Aust J Anat. 2014; 1 (1): 1003.

14. Velló M, Martínez C, Catalá M, Fons J, Salmueras J, Guijarro R. Prenatal and neonatal risk factors the development of enamel defects in low birth weight children. Oral Dis. 2010; 16 (3): 257 62. doi: 10.1111/j.1601-0825.2009.01629.x

15. Enache R, Maxim A, Păsăreanu M. Risk factors involved in the developmental of enamel defects. J Roman Med Dents. 2001; 14 (1): 71-4.

16. Franco KM, Line S, Moura-Rivero M. Prenatal and neonatal variables associated with enamel hypoplasia in deciduous teeth in low birth weight preterm infants. J Appl Oral Sci. 2007; 15: 518-23.

17. Melo N, Vieira R, Soares A. The neonatal intubation causes defects in primary teeth of premature infants. Biomed Pap Med Fac Univ Palacky Olomouc Czech Repub. 2014; 158 (4): 605-12.

18. Machado F, Riberiro R. Defeitos de Esmalte e carie dentaria em crianças premturas e/ou de baixo peso ao nascimiento. Pesqui Bras Odontopediatria Clín Integr. 2004; 4 (3): 243-7.

19. Ferreira F, Ardenghi T.Developmental enamel defects in children prenatally exposed to anti-epileptic drugs. Braz Oral Res. 2011; 25 (6): 531-7.

20. Jayam C, Blandlapalli A, Puttashamachari Y. Developmental enamel defects of primary teeth: a review developmental enamel defects of primary teeth: A review. Glob J Med Res. 2014; 14 (5): 5-7.

21. De la Fuente S. Tablas de contingencia. España: Universidad Autónoma de Madrid; 2011.

22. Robles J. Estudio y prevalencia de los defectos de desarrollo del esmalte en población infantil granadina [Tesis]. Granada: Universidad de Granada, Facultad de Odontodología; 2010.

23. Llopis J. Análisis de correspondencia. La estadística: una orquesta hecha instrumento. 2016. Disponible en https://jllopisperez. com/

24. Jacobsen P. Developmental enamel defects in children born preterm: a systematic review. Eur J Oral Sci. 2014; 121 (1): 1-8. doi: 10.1111/eos.12094 